\title{
Pulmonary perfusion defects in postoperative chest infections
}

\author{
$M$. H. IR VIN ${ }^{1}, R$. A. PAYNE, E. N. O'B RIEN, a nd \\ R. WILKS
}

North Middlesex Hospital, London N.8

The repeated demonstration by many workers that there is a high incidence of asymptomatic postoperative lower limb venous thrombosis suggests that pulmonary embolism is likely to be a more frequent occurrence than is commonly accepted. If this assumption is correct then many emboli must be asymptomatic or producing atypical symptoms.

The incidence of pulmonary artery perfusion defects in 50 general surgical patients who developed postoperative chest complications has been investigated by radioisotope scanning of the lungs. Nine of 10 patients considered on clinical grounds to have had a pulmonary embolus were shown on pulmonary scanning to have perfusion defects which would support such a diagnosis.

Of the $\mathbf{4 0}$ patients diagnosed as having postoperative chest infections, perfusion defects in the lung scan were present in 33. In 19 of these 33 the perfusion defect was associated with an inflammatory lesion or an abnormal position of the diaphragm, visible on the chest radiograph. However, in 7 of the 19 patients the size of the perfusion defect seemed disproportionately large when compared with the extent of the lesion visible on the chest radiograph.

In the remaining 14 patients the perfusion defects were not associated with any abnormality visible on the chest radiograph. It is considered that the perfusion defects in these 14 cases together with those in the seven cases in which the defect was disproportionately large represent 'silent' pulmonary emboli.

It is suggested that pulmonary scanning carried out in cases of persistent postoperative chest infection will reveal, in many instances, a perfusion defect indicative of the presence of an underlying pulmonary embolus. In some cases this will be the precursor of a more major embolus.

Radioisotope scanning of the lungs is now accepted as a sensitive and accurate means of diagnosing pulmonary embolism (Poulose et al., 1970). Errors in interpreting perfusion defects may, however, arise if the scan is assessed without reference to the chest radiograph and clinical findings. Emphysematous bullae, diaphragmatic elevation, bronchial carcinoma, and pulmonary consolidation have all been shown to affect the perfusion pattern (Moser et al., 1966).

Recently there has been a renewal of interest in the problems of venous thrombosis and pulmonary embolism, stimulated by the advent of new diagnostic and therapeutic techniques. Accuracy in detecting lower limb venous thrombi has been improved by the radioactive fibrinogen test, ultrasonic flow measurements, and venography (Flanc, Kakkar, and Clarke, 1968 ; Thomas, McAllister, and Tonge, 1971) while caval

1Present address: Surgical Professorial Unit, St. Bartholomew's Hospital, West Smithfield, London E.C.1 plication, thrombectomy, and thrombolysis have been proved useful in the prophylaxis and treatment of pulmonary embolism (Little and Loewenthal, 1968 ; Paneth, 1970).

These new diagnostic techniques suggest that about one-third of all patients over the age of 40 who undergo a surgical procedure will develop a venous thrombosis, which in about $50 \%$ of cases will be clinically undetectable (Editorial, Lancet, 1970). As a corollary it must be assumed that the incidence of pulmonary embolism is also greater than is clinically apparent.

Necropsy has revealed pulmonary emboli in $51-64 \%$ of unselected cases (Freiman, 1965 ; Morrell and Dunhill, 1968). Andreasen and Lassen (1965) considered pulmonary emboli to be responsible for death in $12.4 \%$ of 890 patients who died following surgery.

It follows that if the incidence of pulmonary embolism is greater than at present clinically diagnosed, it is, in most cases, either asympto- 
matic or being diagnosed as some other condition. Sevitt and Gallagher (1961) observed that postmortem examination of some patients diagnosed in life as having pneumonia revealed pulmonary embolism.

In this study we have attempted to assess, using lung scanning, the incidence of pulmonary embolism in patients diagnosed as having postoperative chest infections and not considered on clinical grounds to be suffering from venous thromboembolic disease.

\section{PATIENTS AND METHOD}

Fifty consecutive cases of postoperative chest complications occurring over a 12-month period among patients on a general surgical unit have been studied. All the patients in the survey were admitted to the adult general surgical wards of one surgeon (R. A. P.) at the North Middlesex Hospital, the majority undergoing major gastrointestinal surgery. Of the 50 patients, 38 were male and 12 female. They represented 1 in 15 of all admissions to the surgical unit. Their ages ranged between 14 and 84, the distribution being shown in Fig. 1, and contrasted with the

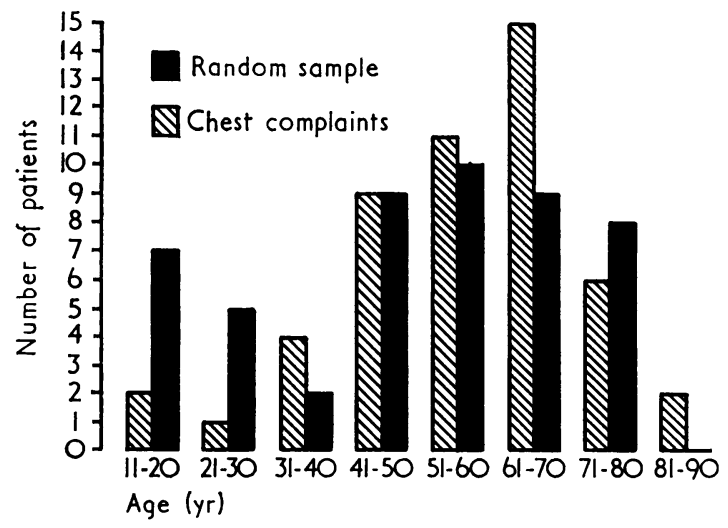

FIG. 1. Age distribution of 50 patients with postoperative chest complications contrasted with the age distribution of a randomly selected sample of patients admitted to the same wards.

age distribution of a randomly selected group of 50 patients admitted to the wards over the same period. The patients were grouped on the basis of clinical examination into those diagnosed as having "chest infections' and those diagnosed as having 'pulmonary embolism'.

A chest infection was defined as the presence in a patient on or after the fourth postoperative day of a combination of cough, pyrexia, purulent sputum, and adventitious sounds in the lungs. The choice of the fourth postoperative day as the reference point was designed to exclude those patients with minor postanaesthetic respiratory symptoms and pyrexial reactions which could be expected to subside during the first 48 hours after operation.

No distinction was made for the purpose of inclusion in the survey between those patients with bronchitis and no, or minimal, changes on chest radiography and those with clinical and radiological evidence of consolidation or atelectasis, though these factors were taken into account when the scans were assessed.

The definition of what clinical features should be accepted as diagnostic of pulmonary embolism proved more difficult. For the purpose of this study we have regarded the combination of pleuritic pain and frank haemoptysis as diagnostic, irrespective of whether or not there was associated dyspnoea, cyanosis or evidence of right ventricular strain. The massive type of pulmonary embolism which causes sudden collapse and profound hypotension was not encountered as a presenting feature in this series.

The presence of clinical signs indicative of lower limb venous thrombosis was accepted as indicating that pulmonary complications of a non-specific nature were likely to be the result of pulmonary embolism and such cases were thus included in the latter group. The incidence of lower limb thrombosis was not assessed other than clinically.

Upon inclusion in the survey patients had a chest film and lung scan within 24 hours following which appropriate antibiotic or anticoagulant treatment was instituted. Radiographs and scans were taken under optimum conditions in the appropriate department. Where possible patients whose scan showed a perfusion defect had a further chest radiograph and scan three months after the first scan together with pulmonary function tests if chronic respiratory disease was suspected. Although preoperative lung function testing would have been of value in assessing the lung scans, the work load involved in assessing all patients admitted for surgery made this impracticable. Similarly, it would have been informative to have lung scans on postoperative patients with no apparent chest complications, and to have pulmonary angiography in those patients with chest infections who were shown on scanning to have perfusion defects. However, neither procedure was felt to be ethical.

It is the practice in this hospital for the chest films and scans to be reported on separately, the latter by a physicist who provides a factual description of the perfusion defects present in the scan. In this study films and lung scans were initially reported upon in the usual manner and were subsequently reassessed by a combined group consisting of a chest physician, physicist, and surgeon.

SCANNING TECHNIQUE Prior to scanning the thyroid was blocked by giving $400 \mathrm{mg}$ potassium perchlorate. The patient, positioned supine, received an intravenous injection of $100 \mu \mathrm{Ci}$ of macroaggregated albumin labelled with iodine-131 (131I) with a particle size ranging from 10 to 50 microns. Scanning began immediately after injection. 
A Picker magnascanner with a 3 inch diameter $\mathrm{NaI}$ (Th) crystal was used with its standard 19 hole focussing collimator accepting the pulses straddling the photopeak of the $360 \mathrm{keV}$ ray of ${ }^{131} \mathrm{I}$ in a channel width of approximately $55 \mathrm{keV}$.

The scanning head was positioned over the point of maximum count rate from the patient's lungs and the rate meter was adjusted to give between 95 and $100 \%$ of full-scale deflection. The rate meter time constant selected was such that at maximum count rate the standard deviation corresponded to $1 / 16$ th of a full-scale deflection (half a colour change of the 8 colour printer). With this time constant the scanning speed was adjusted to ensure that $86 \%$ of any change in count rate would be attained while traversing a lesion of diameter $1.5 \mathrm{~cm}$. Scanning then began with the machine being used in the unidirectional and constant dot spacing modes (Wighton and Mackenzie, 1968). The scanning procedure lasted about 40 minutes. Although posterior and lateral scans were carried out where indicated only the anterior scan was routinely obtained as it was felt unjustified to obtain more than this in patients after major abdominal surgery.

\section{RESULTS}

PREOPERATIVE CHEST CONDITION At routine preoperative examination only eight patients gave a history of significant respiratory disease. In only five of these was this associated with abnormal physical signs in the chest and abnormalities visible on the preoperative chest radiograph.

POSTOPERATIVE CHEST CONDITION Of the 50 patients in the survey, 40 were classified, on clinical grounds, as having chest infections and 10 as having pulmonary embolism. The incidence of reported perfusion defects in the scans, and the incidence of abnormality visible on the chest radiographs in each of the two groups, is shown in the Table.

THE COMBINED ASSESSMENT Reassessment of the scans and films by the combined group confirmed the presence of the perfusion defects reported by the physicist in all the pulmonary embolism cases and in all but one of the chest infection cases.

\section{T A B L E}

INCIDENCE OF PERFUSION DEFECTS IN LUNG SCANS, AND OF RADIOLOGICAL CHANGES IN CHEST FILMS, OF 50 PATIENTS WITH POSTOPERATIVE CHEST COMPLI-

\begin{tabular}{|c|c|c|c|}
\hline & & $\begin{array}{l}\text { Pulmonary } \\
\text { Embolism } \\
\text { (10 cases) }\end{array}$ & $\begin{array}{c}\text { Chest } \\
\text { Infection } \\
\text { (40 cases) }\end{array}$ \\
\hline $\begin{array}{l}\text { Pulmonary scan defect } \\
\text { Normal scan } \\
\text { Chest } x \text {-ray lesion } \\
\text { Normal chest } x \text {-ray }\end{array}$ & $\begin{array}{l}\because \\
\because \\
\because\end{array}$ & $\begin{array}{r}9 \\
1 \\
10 \\
0\end{array}$ & $\begin{array}{r}34 \\
6 \\
21 \\
19\end{array}$ \\
\hline
\end{tabular}

Perfusion defects observed in the scans of the patients in the pulmonary embolism group were of the type expected and similar to those reported by other workers (Fig. 2). All the pulmonary embolism cases had some abnormality on chest radiography but in only three cases were the abnormalities indicative of pulmonary embolism.

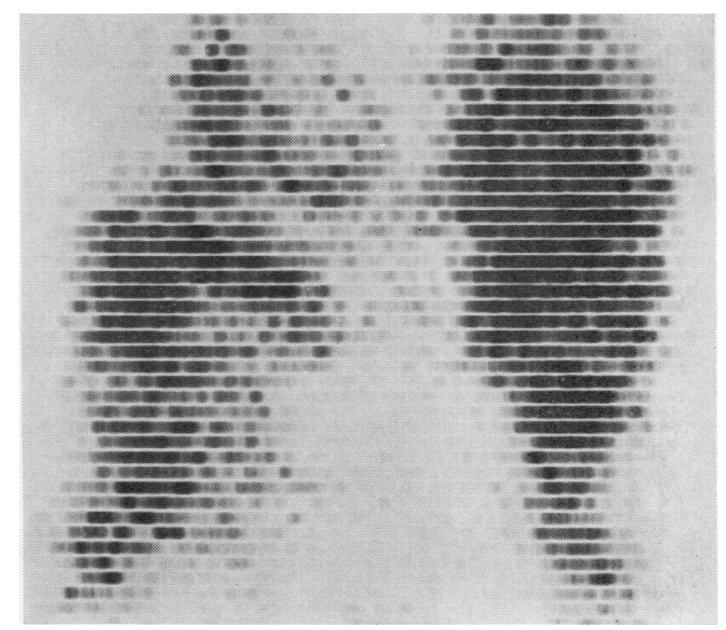

FIG. 2. Lung scan in patient with clinical diagnosis of pulmonary embolism. Scan shows multiple crescentic defects in the right lung compatible with pulmonary embolism.

Of the 33 chest infection cases with perfusion defects, 19 had an abnormality visible on the postoperative chest film. In four of these the radiograph showed that an apparent lower lobe perfusion defect was due to a raised hemidiaphragm. The remaining 15 had evidence of inflammatory disease associated with the perfusion defect, but in seven of these the extent of the perfusion defect seemed out of proportion to the degree of pathology demonstrated both clinically and radiologically.

The history of one of these cases, though atypical in that the patient was the only one in the series who died before discharge from hospital, indicates the possible significance of the perfusion defects.

Case history A. T., a 54-year-old man, was admitted with a carcinoma of the rectum. Examination before operation revealed a fit-looking man with an arterial blood pressure of $150 / 80 \mathrm{mmHg}$.

There was no history of pulmonary disease and the only abnormality detectable on examination of the chest was some bronchospasm in the right mid 


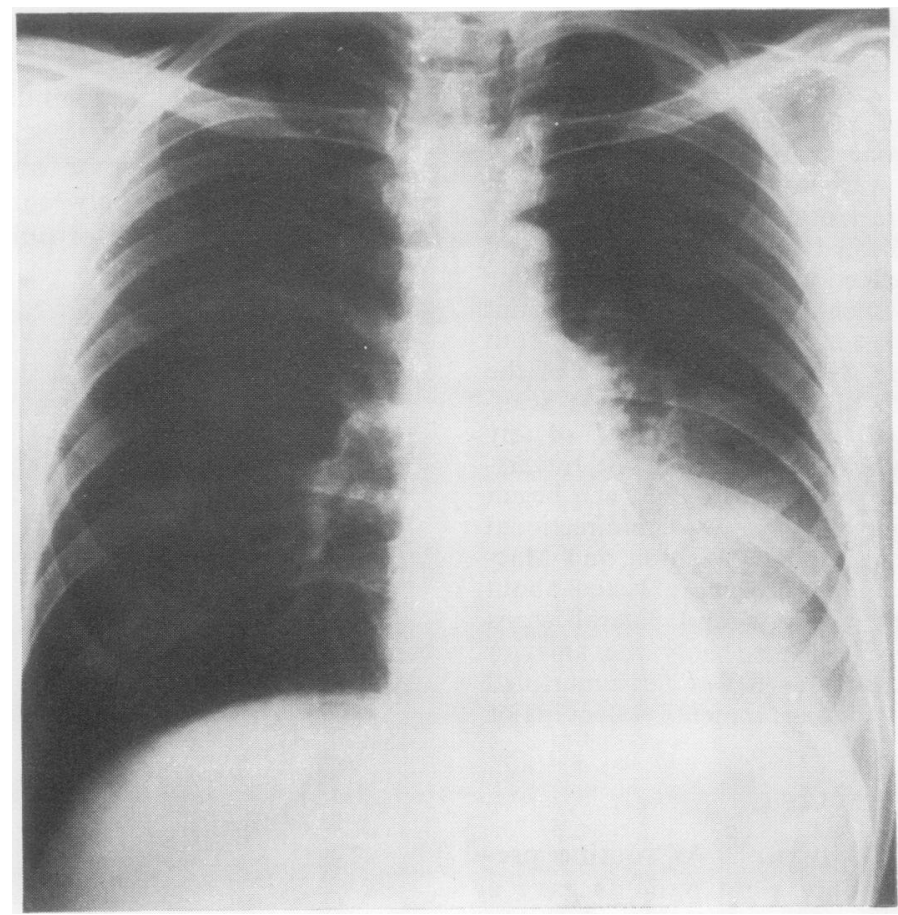

FIG. 3. Postoperative chest radiograph of patient with clinical chest infection showing consolidation of left lower lobe.

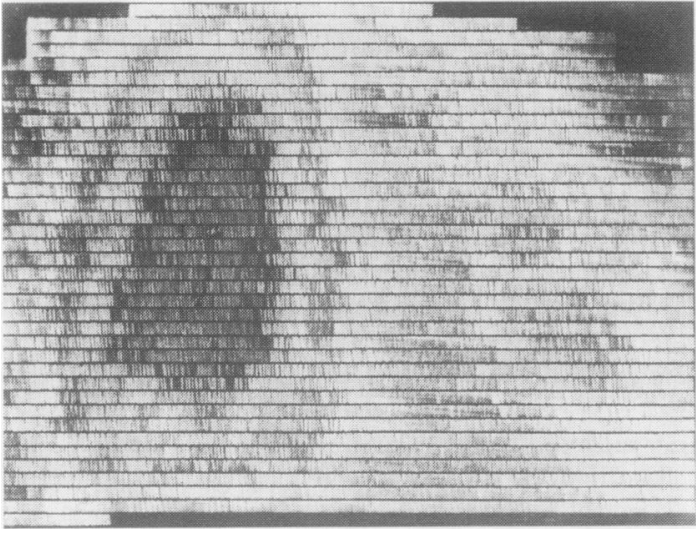

FIG. 4 Lung scan in same patient as in Fig. 3 showing absence of isotope uptake in left lung (black/white print from colour scan).

zone. The preoperative chest radiograph, haemoglobin, and white cell count were normal.

Routine abdominoperineal excision of the rectum was carried out, no evidence of metastases being observed. On the fourth postoperative day he developed a cough with purulent sputum and a pyrexia of $38.3^{\circ} \mathrm{C}$. Physical examination revealed dullness to percussion and bronchial breathing at the left base.

A chest infection was diagnosed and treatment with ampicillin was begun. Sputum culture showed a growth of Streptococcus pneumoniae. The chest radiograph at this time showed consolidation of the left lower lobe (Fig. 3). The pulmonary scan, however, showed virtually no uptake in the left lung (Fig. 4) and was considered diagnostic of pulmonary embolism. At no time did the patient show any signs of lower limb venous thrombosis although four days after the onset of his respiratory symptoms he had a small haemoptysis.

Three days later he burst his abdominal wound and needed a further anaesthetic for resuture. Ten days later he collapsed and died with an obvious major pulmonary embolus. Necropsy showed the presence of a large fresh embolus impacted in the right ventricle, an adherent embolus in the left pulmonary artery, and infarction of the left lower lobe.

The findings in 14 patients of perfusion defects that were not associated with radiological abnormality are particularly significant. In the majority the perfusion defects were similar to those seen in the pulmonary embolism cases and in many instances were more extensive.

In two patients an abnormality visible on the 
chest radiograph was not accompanied by a perfusion defect while five patients had neither radiological change nor scan defect.

PROGRESS OF PATIENTS Where possible patients shown to have perfusion defects had a further radiograph and pulmonary scan three months after their initial investigations. Of the pulmonary embolism cases two died from their underlying malignant condition. In half the remainder the perfusion defects resolved while in the other half the defects were still evident.

The picture was similar in the chest infection group. Four died, two from their underlying carcinoma and two from 'pneumonia' some 8 to 10 weeks after operation for benign conditions. In the remainder the picture was similar to that of the pulmonary embolism group in that in half the perfusion defects resolved whereas in the other half they were still evident.

\section{DISCUSSION}

Although signs of pulmonary embolism, such as lobe shrinkage, localized areas of narrowing of the pulmonary vessels, opaque shadowing and effusion, and right heart dilatation, may be seen on the plain chest radiograph (Simon, 1971) radiography is considered to be an unreliable method by which to diagnose pulmonary embolism.

The most reliable method of diagnosing occlusion of the pulmonary arteries is angiography but this procedure is not without hazard to the patient and is only justified if the information obtained will materially affect the treatment.

The experimental demonstration in 1964 by Sabiston and Wagner that radioisotope scanning of the lungs would reveal absence of isotope uptake in any area of the lung which had its arterial supply occluded was thus particularly significant. The further observation by Moser et al. (1969) of a high degree of correlation between the isotope scan and the pulmonary angiogram further enhanced the potential value of the scan.

Although studies by Walker and Jackson (1969) showed the combined use of pulmonary angiography and radioisotope scanning led to a greater degree of detection of pulmonary emboli than either method alone, it is now accepted that for all practical purposes the isotope scan is almost as accurate as the angiogram provided it is carefully interpreted (Poulose et al., 1970).

In this present investigation 9 of the 10 cases of clinically diagnosed pulmonary embolism were shown to have characteristic perfusion defects on the pulmonary scan. In addition 33 of the 40 cases of chest infection were also shown to have perfusion defects, the cause of which in many cases was not readily apparent. In this context it is important to recall the basic observation by Walker and Jackson (1969) that an area in which there is no isotope retention is not perfused with blood. There are, however, numerous reasons for such a lack of perfusion.

In four cases in the present series an apparent lower lobe perfusion defect was accounted for by a raised hemidiaphragm. Other obvious external causes such as pleural effusion, emphysematous bullae, or thoracic cage trauma were not encountered.

The effect of inflammatory lesions on the pulmonary scan is not well documented. In the present series 15 had radiologically visible inflammatory lesions in the area of the perfusion defect, but in seven of these the defect appeared disproportionately large when compared with the size of the inflammatory lesion. On the other hand, two patients with inflammatory lesions had no scan defect.

Ormond, Drake, Rideout, and Misra (1969) pointed out that parenchymal consolidation will produce a scan defect, and Fred et al. (1966) was able to demonstrate perfusion defects seen in the scan in cases of pneumonia and left ventricular failure which could not be confirmed by angiography. However, Walker and Jackson (1969) commented that such defects were never as marked as in pulmonary embolism. It is likely that the early stages of lobar pneumonia are in fact associated with hyperaemia (Silver, 1968). Rosenthal (1965) demonstrated the presence of normal perfusion in four cases of bronchopneumonia.

Where perfusion defects occur due to a cause other than pulmonary embolism Poulose et al. (1970) consider that they can be differentiated by considering the shape of the scan defect and contrasting it with the segmental or crescentic type of lesion usually caused by a pulmonary embolus. Similarly, Silver (1968) commented that even though large emphysematous bullae could produce localized ischaemia they seldom, if ever, produced a lobar or segmental artery obstruction. In our series the nature of the scan defect in most of the 21 cases with unexplained or disproportionate defects was in keeping with an obstruction in the pulmonary artery.

Although it is usually assumed that the cause of obstruction of the pulmonary artery in postoperative patients is embolic, disseminated intravascular coagulation in patients who have been subject to extensive surgical trauma or prolonged 
hypotension must also be considered as a cause of pulmonary arteriolar occlusion, producing multiple scattered defects on the pulmonary scan.

It must thus be concluded that the cause of the perfusion defects in those patients in whom there was no other obvious explanation was in fact pulmonary embolism from a peripheral venous thrombosis, and that the effect of the embolus upon the lung parenchyma was such as to predispose it to the development of infection. It is very unlikely that a significant perfusion defect will result from lung collapse, mucus plugs or consolidation in cases where the changes induced are insufficient to be visible radiologically.

Although major pulmonary embolism is usually assumed to cause considerable systemic upset with dyspnoea, hypotension, and right ventricular strain, the condition may be unaccompanied by any appreciable disturbance of cardiorespiratory function or abnormality in the electrocardiogram or chest film. Parmley, Senior, McKenna, and Johnston (1970) have described four such cases with massive pulmonary embolism in which the pulmonary scan revealed large perfusion defects but which clinically were unaccompanied by any of the usual signs.

That unexplained perfusion defects in the present cases are the result of pulmonary embolism raises fresh factors in the management of postoperative chest complications. In patients in whom the scan confirms the clinical impression of pulmonary embolism the clinician will treat the case in the manner to which he is accustomed, that is, with anticoagulants supplemented occasionally by caval ligation and thrombolytics.

On the other hand, the discovery of an otherwise inexplicable perfusion defect in a patient who apparently has only a chest infection poses a considerable dilemma, especially if the infection appears to be resolving on treatment with antibiotics. There would be a reluctance to use anticoagulants in such circumstances for most clinicians are acutely aware of the many complications of such therapy. However, as Parmley et al. (1970) have demonstrated, such perfusion defects, if the result of pulmonary embolism, may herald a major embolus later. The proven occurrence of one such episode in the present series together with the unexplained death from 'pneumonia' of two patients who were known to have had perfusion defects indicates the probable risk in such cases.

It is suggested that established chest infection occurring in a patient after surgery may indicate the presence of an underlying pulmonary embolus.
Pulmonary scanning is indicated in any patient who, in the postoperative period, develops other than a transient chest infection. If a significant perfusion defect is found for which there is no obvious explanation on the chest film the patient should be regarded as having had a pulmonary embolus and investigated further by venography, ultrasonic flow measurements, and pulmonary angiography.

This study was carried out within the confines imposed by a busy general surgical unit in a regional hospital. It suggests the need for further investigation of the association between chest infections and pulmonary embolism.

\section{REFERENCES}

Andreasen, C., and Lassen, H. K. (1965). Fatal pulmonary embolism in a surgical department during a period of fifteen years. Acta chir. scand., Suppl. 343, 42.

Editorial (1970). Lancet, 1, 395.

Flanc, C., Kakkar, V. V., and Clarke, M. B. (1968). The detection of venous thrombosis of the legs using ${ }^{125} \mathrm{I}$ labelled fibrinogen. Brit. J. Surg., 55, 742.

Fred, H. L., Gonzalez, D. A., Lockhart, R. W., Peabody, C. A., and Alexander, J. K. (1966). Selective arteriography and lung scanning in the differential diagnosis of pulmonary thromboembolism (abstract). Clin. Res., 14, 365.

Freiman, D. G. (1965). Pathologic observations on experimental and human thromboembolism, In Pulmonary Embolic Disease, edited by A. A. Sasahara and M. Stein. Grune and Stratton, New York.

Little, J. M., and Loewenthal, J. (1968). Factors contributing to a lowered mortality from pulmonary embolism. Surg. Gynec. Obstet., 127, 777.

Morrell, M. T., and Dunnill, M. S. (1968). The post-mortem incidence of pulmonary embolism in a hospital population. Brit. J. Surg., 55, 347.

Moser, K. M., Harsanyi, P., Garriga, G. R., Guisan, M., Landis, G. A., and Miale, A. (1969). Assessments of pulmonary photo scanning and angiography in experimental pulmonary embolism. Circulation, 39, 663.

, Rhodes, P. G., Landis, G., Tisi, G., and Miale, A. (1966). Interpretive pitfalls in lung scanning (abstract). J. Nucl. Med., 7, 353.

Ormond, R. S., Drake, E. H., Rideout, R. A., and Misra, G. (1969). Pulmonary angiograms and isotope lung scansrole in the diagnosis of pulmonary embolism. Henry Ford Hosp. Medicine Journal, 17, 3.

Paneth, M. (1970). Surgical management of massive pulmonary embolism. Brit. med. J., 2, 778.

Parmley, L. F., Senior, R. M., McKenna, D. H., and Johnston, G. S. (1970). Clinically deceptive massive pulmonary embolism. Chest, 58, 15.

Poulose, K. P., Reba, R. C., Gilday, D. L., Deland, F. H., and Wagner, H. N. (1970). Diagnosis of pulmonary embolism. A correlative study of the clinical, scan, and angiographic findings. Brit. med. J., 3, 67. 
Rosenthal, L. (1965). Lung scanning with radioiodinated macroaggregates of human serum albumin. J. Canad. Ass. Radiol., 16, 30.

Sabiston, D. C., and Wagner, H. N. (1964). The diagnosis of pulmonary embolism by radioisotope scanning. Ann. Surg., 160, 575.

Sevitt, S., and Gallagher, N. (1961). Venous thrombosis and pulmonary embolism. Brit. J. Surg., 48, 475.

Silver, S. (1968). Lung function including blood flow studied with radioactive isotopes: lung scans. Radioactive Nuclides in Medicine and Biology, 3rd ed., p. 444. Lea and Febiger, Philadelphia.
Simon, G. (1971). Principles of Chest X-Ray Diagnosis, 3rd ed. Butterworth, London.

Thomas, M. L., McAllister, V., and Tonge, K. (1971). Simplified phlebography in deep venous thrombosis. Clin. Radiol., 22, 490.

Walker, R. H. S., and Jackson, J. A. (1969). Scanning of the lung. Proc. roy. Soc. Med. 62, 799.

Wighton, R. J., and Mackenzie, L. J. (1968). The Picker Magna scanner with constant dot spacing colour printout and background cut-off. Brit. J. Radiol., 41, 875. 